\title{
Análisis de la resistencia de piezas soldadas por el proceso de resistencia eléctrica
}

\section{Analysis of the resistance of welded parts by the electrical resistance process}

\author{
MEDINA-MENDOZA, José Antonio†*, MÉNDEZ-MACÍAS, Gerardo y HERRERA-REYES, Naieli \\ Universidad Tecnológica del Norte de Aguascalientes, Departamento de Ingenierías, Aguascalientes, México.
}

ID $1^{\mathrm{er}}$ Autor: José Antonio, Medina-Mendoza / ORC ID: 0000-0002-3969-5765, CVU CONACYT ID: 522777

ID $1^{\text {er }}$ Coautor: Gerardo, Méndez-Macías / ORC ID: 0000-0001-9141-656X, CVU CONACYT ID: 645250

ID $2^{\text {do }}$ Coautor: Naieli, Herrera-Reyes / ORC ID: 0000-0002-7654-1167, CVU CONACYT ID: 564450

DOI: $10.35429 /$ JEE.2020.13.4.1.9

Recibido Julio 10, 2020; Aceptado Diciembre 30, 2020

\section{Resumen}

Se ha realizado una estancia en una empresa metalmecánica ubicada en el Parque Industrial del Valle de Aguascalientes, con la finalidad de desarrollar un estudio que pueda reflejar el estado de la fabricación de diversas piezas metálicas que para su desarrollo implican un proceso de soldadura por resistencia eléctrica. El área de fabricación es denominada Automotriz y cuenta con 5 máquinas de soldadura de diversas marcas y capacidades, en las cuales se fabrican modelos diferentes de piezas automotrices para diversos clientes de la industria automotriz en el estado de Aguascalientes, México. El estudio se enfoca en la revisión del proceso basándose en las normatividades que se manejan en la empresa, que son las JIS B 1196 referentes a la soldadura por puntos. De esta normatividad se extraen dos características principales para garantizar la calidad del producto: la resistencia a la tensión de la soldadura y la resistencia a la torsión. Las normas de fabricación utilizadas determinan que cada 2 horas hay que realizar prueba de tensión en las diferentes partes manufacturadas según su clasificación, se prueba la soldadura en tuercas 6M, 8M Y 10M, designadas por el sistema internacional aplicándoles cargas en una prensa hidráulica marca POISE modelo DX-25 y cien por ciento pruebas de torsión en piezas que llevan soldadas tuercas $6 \mathrm{M}$, las cuales son probadas en un dispositivo de diseño propio. Las tomas de datos para la evaluación se obtuvieron en el laboratorio de metrología de la empresa y muestran una dispersión muy marcada, aunque dichos datos no rebasan en apariencia los límites establecidos por la empresa. Se llevó a cabo una revisión y evaluación del proceso y se propusieron los límites del ajuste de resistencia del proceso de soldadura y las mejoras que garanticen una buena calidad de los productos. Se encontraron algunas prácticas que pueden afectar la calidad, se describen y se propone una solución para evitar que se realicen en el proceso.

Soldadura por resistencia eléctrica, Normas JIS B 1196
Abstract

A stay has been carried out in a metalworking company located in the Valle de Aguascalientes Industrial Park, in order to carry out a study that can reflect the state of manufacture of various metal parts that involve an electrical resistance welding process. The manufacturing area is called Automotive and has 5 welding machines of various brands and capacities in which different models of automotive parts are manufactured for various clients of the automotive industry in Aguascalientes Mexico. The study focuses on the review of the process based on the regulations that are handled in the company and that are the JIS B 1196 referring to spot welding, from which two main characteristics are extracted to guarantee the quality of the product and that are the resistance to weld stress and torsional strength. The manufacturing standards used determine that every hour a stress test must be carried out on the different manufactured parts according to their classification, welding is tested on $6 \mathrm{M}, 8 \mathrm{M}$ and $10 \mathrm{M}$ nuts of the international system applying loads in a brand FORSE DX-2.5 model hydraulic press and one hundred percent tests torque on parts with $6 \mathrm{M}$ nuts welded, which are tested in a devise of our own design. The data taken for the evaluation were obtained in the company's metrology laboratory and showed a very marked dispersion, although said data apparently did not exceed the established limits. A review and evaluation of the process was carried out and limits and improvements were proposed that guarantee good quality of the products. Some practices that can affect quality were found, they are described and a solution is proposed to prevent them from being carried out in the process.

Welding, Regulations JIS B 1196

Citación: MEDINA-MENDOZA, José Antonio, MÉNDEZ-MACÍAS, Gerardo y HERRERA-REYES, Naieli. Análisis de la resistencia de piezas soldadas por el proceso de resistencia eléctrica. Revista de Ingeniería Eléctrica. 2020. 4-13:1-9.

$\dagger$ Investigador contribuyendo como primer autor. 


\section{Introducción}

Desde la década de los 80's se han observado políticas de crecimiento en el estado de Aguascalientes, teniéndose una tendencia elevada en el desarrollo de estas políticas desde ese tiempo y que han contribuido al desarrollo concentración de actividades productivas en el estado.

Esta actividad ha transformado el mercado Industrial y a la población, desarrollando nuevas formas de consumo cultural.

Es importante mencionar que el estado de Aguascalientes ingresó a una economía global, y la llegada de grandes empresas transnacionales transformó su fisonomía y genero cambios estructurales a su economía, manteniendo una participación creciente en el comercio internacional.

El nivel tecnológico que se ha alcanzado el estado de Aguascalientes, lo ha llevado a un desarrollo regional importante que ha integrado a diferentes empresas en diferentes niveles y son, la empresa automotriz, agroindustrial, metalmecánica, electrónica, textil y del vestido.

La inversión extranjera en Aguascalientes proviene básicamente de dos países, Estados Unidos de América y Japón. Está inversión se centran en un sector principal, la manufactura de partes metálicas, maquinaria y equipo en donde se clasifica el sector automotriz. El estado de Aguascalientes se apuntala como el principal beneficiario de capitales japoneses y cuenta con el $32 \%$ de la inversión en México e integra 46 empresas en diferentes sectores que operan en el territorio estatal, así también, generan 17 mil empleos directos y de planta. Estos grandes proyectos industriales favorecen el crecimiento de nuevos distribuidores y cadenas de suministro hasta de $5^{\text {a }}$ generación.

En este entorno de crecimiento, la empresa en la que se realizó el presente estudio es fundada en el año 2006. Es una empresa orgullosamente mexicana que cuenta con más de 12 años de trayectoria siendo líder en manufactura y comercialización de productos metálicos de la más alta calidad.
La empresa en cuestión ofrece soluciones a un gran número de mercados mediante distintas líneas de negocio. Se ha posicionado como líder en el diseño y manufactura de partes automotrices y comercialización de productos metálicos para diferentes aplicaciones. Apostando por la innovación continua en tecnología y desarrollo, pero, sobre todo, apoyándose del factor humano que es el motor para desarrollar nuevos proyectos enfocados en ofrecer soluciones metálicas eficientes.

En el año 2013, la empresa se unió con más de 20 empresas importantes del estado para formar un grupo nuevo, Grupo Industrial Automotriz MAEN (Manufacturing Automotive Enterprises Network) con la finalidad de unir esfuerzos y proyectar su trabajo con mayor eficacia, compartiendo experiencias y complementándose en su capacidad de producción y desarrollo. Este nuevo grupo posee los mejores procesos de producción: sheetmetal, corte laser, estampado, doblado de tubo, formado de alambre, fibra de vidrio, inyección de plástico, extrusión de plástico, ensambles de soldadura, pintura electrostática, galvanizado electrolítico, automatizaciones y mecanizaciones, entre otros. La principal meta es satisfacer las necesidades industriales para pequeñas y medianas empresas, así como para corporativos internacionales.

La empresa cuenta con un área de maquila metálica que se denomina automotriz, en la cual se manufacturan alrededor de 30 diferentes partes automotrices de soportería y es en esta en donde se centran las bases de este estudio. La empresa cuenta con 10 máquinas de soldadura por resistencia eléctrica y de las cuales durante el tiempo en que se realizó la evaluación del proceso se trabajaba en 5 para soportería y 2 para cubierta de múltiple de admisión de motor. El estudio plantea una revisión de la calidad de la soldadura en las partes de soportería automotriz comparando con las normas establecidas emanadas de la norma JIS 1186 2010.

\section{Justificación}

Las piezas que se fabrican en la empresa, dentro del proceso de soldadura, son sometidas a dos pruebas básicas y que están relacionadas entre sí. 
Una prueba corresponde al ensayo destructivo de tensión, que se aplica para despegar las tuercas soldadas de las láminas troqueladas correspondientes a los diferentes soportes metálicos; y el otro, un ensayo no destructivo correspondiente de someter a una fuerza de torsión en las tuercas soldadas. Los datos de medición de las pruebas de tensión muestran una dispersión muy amplia, por lo que es necesario realizar un estudio para comparar con la norma establecida o de ser necesario, establecer una norma diferente para este proceso de soldadura. Para lo cual es necesario, revisar la norma establecida para la prueba de torsión y verificar que se esté aplicando con los parámetros establecidos de acuerdo con la norma.

\section{Objetivo}

Establecer las normas adecuadas en los procesos de soldadura por puntos para garantizar que los límites superior e inferior de la resistencia a la tensión y torsión sean los adecuados para que no se presenten fallas durante los ensambles finales de las piezas manufacturadas.

\section{Marco teórico}

Siempre es difícil soldar láminas pequeñas y delgadas, o materiales metálicos diferentes con un proceso de soldadura al arco eléctrico, también puede ser difícil con un proceso de soldadura con plata o estaño, por esta razón es conveniente disponer de una soldadura por puntos. La presión y temperatura son los principales factores para lograr una soldadura por puntos, cuando por dos electrodos en forma de pinza se hace pasar una corriente eléctrica y se logra una temperatura próxima a la fusión, al presionar precisamente con las pinzas, las piezas se sueldan entre sí (Figura 1).

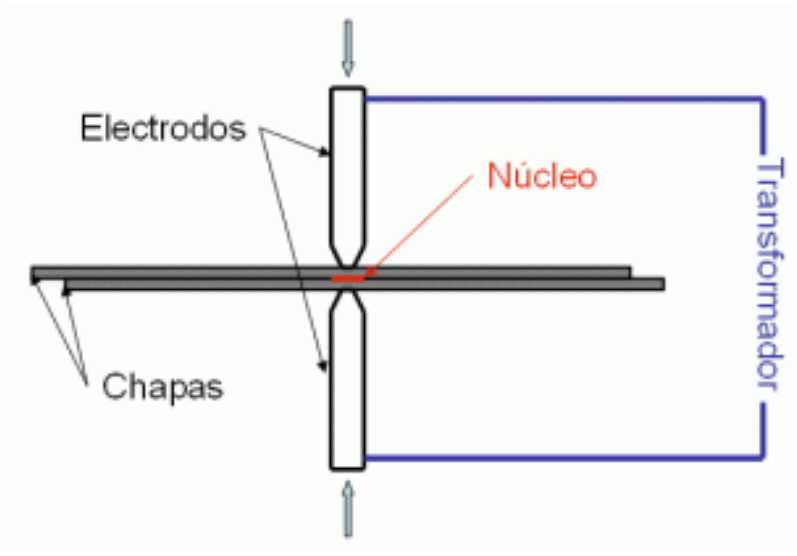

Figura 1 Diagrama del pegado por puntos

ECORFAN $^{\circledR}$ Todos los derechos reservados.

Debido a que generalmente la resistencia al paso de la corriente de las piezas a soldar es muy baja, la corriente que debe pasa por la zona a soldar debe ser muy alta del orden de los 500 amperios, pero sin embargo los voltajes son muy bajos, de 1 a 3 voltios. La potencia total es por tanto uno o dos kilovatios.

Los electrodos tienen la misión de presionar los metales y enseguida hacer pasar la corriente a través de las piezas metálicas a soldar. Sus puntas deben estar alineadas al acercarse y deben estar aislados entre sí. Los electrodos deben estar muy bien conectados con el secundario del transformador.

\section{Fases de la soldadura por puntos}

1. Colocar las piezas a soldar entre las pinzas.

2. Bajar los electrodos, que corresponde al tiempo que transcurre desde la operación de acercamiento hasta que comienza el paso de la corriente.

3. Tiempo de soldadura, que consiste en el tiempo durante el cual está pasando la corriente eléctrica.

4. Tiempo de forja, es el tiempo transcurrido entre el corte de la corriente y el levantamiento de los electrodos.

5. Tiempo de enfriamiento, consiste en la desaparición de la presión en las piezas soldadas (Figura 2).

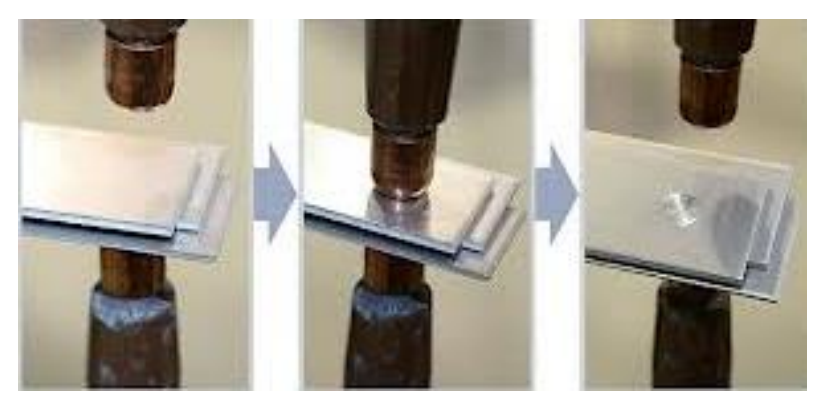

Figura 2 Fases de la soldadura por puntos

El proceso de soldadura por puntos tiende a endurecer el material, hacer que se deforme, reducir la resistencia a la fatiga del material, y puede estirar el material. Los efectos físicos de la soldadura por puntos pueden crear fisuras internas y grietas en la superficie. Las propiedades químicas afectadas son la resistencia interna del metal y sus propiedades corrosivas.

MEDINA-MENDOZA, José Antonio, MÉNDEZMACÍAS, Gerardo y HERRERA-REYES, Naieli. Análisis de la resistencia de piezas soldadas por el proceso de resistencia eléctrica. 2020 
Éste es el sistema de soldadura que se emplea con mayor frecuencia tanto en fabricación como en reparación, debido a las ventajas que posee frente a otros sistemas de unión. Siempre que su ejecución se lleve a cabo de la manera correcta, se obtienen soldaduras de buena calidad y uniformes, que presentan una zona fundida homogénea, sin huecos, rechupados o grietas.

Su manejo es sencillo, ya que la mayoría de los equipos utilizados hoy en día, tanto en fabricación como en reparación, son automáticos (Figura 3). Por esta razón, la calidad final ya no depende tanto de la destreza del operario, sino de la regulación de los parámetros de la máquina de acuerdo con el tipo de trabajo que se desea realizar.

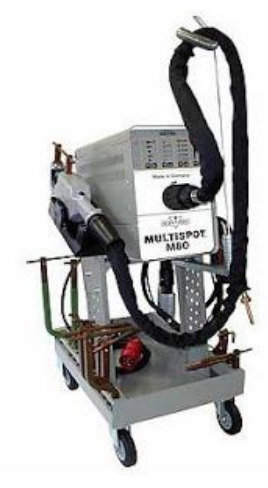

Figura 3 Equipo para soldadura por puntos manual

Algunas de las ventajas de este sistema son:

Ausencia de deformaciones y cambios en la estructura del material, debido a que la aplicación de calor es mínima y se lleva a cabo de forma muy localizada en la zona de contacto de los electrodos.

- Las superficies que se obtienen son relativamente suaves, libres de fusión superficial o huellas profundas, que hacen innecesario un repaso posterior como operación de acabado.

No requiere material de aportación, lo cual reduce costos.

El desmontaje de piezas unidas por puntos de resistencia es sencillo. Haciendo uso de las herramientas específicas para esta función, se realizará de forma rápida y, lo que es aún más importante, sin causar desperfectos en las piezas adyacentes.

\section{Parámetros de la soldadura.}

Este proceso depende directamente del tiempo y el paso de la corriente eléctrica para obtener el calor necesario y lograr un estado pastoso de las láminas a soldar. Debido a esto, estas dos variables se tienen que regular adecuadamente. Para obtener una soldadura rápida se puede aumentar la intensidad de la corriente y disminuir el tiempo de soldadura, o bien se puede optar por un aumento de tiempo y disminuir la intensidad de corriente obteniendo una denominada soldadura lenta. Estas variaciones oscilan dentro de unos márgenes determinados, pues en la práctica no es posible disponer de tiempos ni de intensidades tan amplias como se desee. La intensidad máxima que se quiere alcanzar está condicionada por el equipo concreto con el que se ejecute la soldadura y por las piezas que se van a unir, ya que hay que evitar su fusión. El tiempo de soldadura no debe ser muy prolongado, ya que parte del calor se perdería por conducción y se puede llegar a establecer un equilibrio térmico entre calor aportado y calor disipado, que impediría alcanzar una temperatura suficiente y daría lugar a uniones de escasa o nula resistencia mecánica. Para lograr un buen punto de soldadura se debe:

- Seleccionar la intensidad máxima del equipo, sin que se llegue a producir la fusión de las láminas.

- Regular el tiempo de paso de la corriente en función del espesor que se pretende soldar.

\section{Resistencia eléctrica de la unión.}

La resistencia eléctrica de la unión no es un parámetro que pueda ser regulado en el equipo utilizado en el proceso de soldadura y depende de la naturaleza del material.

La resistencia que opone un material al paso de la de la corriente es inversamente proporcional a su conductividad eléctrica, dependiendo fundamentalmente de su composición y de su tratamiento, así como de su acabado y limpieza. Es un factor que se debe tener en cuenta, ya que la resistencia influye directamente en la cantidad de calor generada durante la soldadura. 
Por tanto, a mayor conductividad eléctrica, menor resistencia al paso de la corriente, hecho que dificulta la realización de este tipo de soldaduras. Este inconveniente se puede subsanar con un aumento de la intensidad de la corriente.

\section{Presión de apriete.}

La soldadura por puntos de resistencia es una soldadura por forja, lo cual supone la existencia de una conformación en caliente, que se obtiene mediante la presión aplicada a través de los electrodos. A este parámetro no se le suele dar la importancia que merece, que se supone de manifiesto en dos acciones distintas: su influencia sobre la resistencia y su efecto de forja durante la solidificación del núcleo de metal fundido.

La aplicación de la presión tiene tres misiones distintas, que pueden parecer incluso contradictorias:

1. En el momento de comenzar la soldadura, la presión ha de ser baja para obtener una resistencia de contacto chapa-chapa elevada, que permita un calentamiento inicial con intensidad moderada.

2. A su vez, esta presión ha de ser suficiente para que las chapas a unir tengan un contacto adecuado y se acoplen entre sí perfectamente.

3. Una vez que se ha iniciado la fusión del punto, la resistencia de contacto queda reducida a la zona delimitada por los electrodos. En ese momento, la presión debe ser alta para expulsar los gases incluidos y llevar a cabo la forja del punto.

4. La presión recomendada para la chapa de acero se halla alrededor de los $10 \mathrm{~kg} / \mathrm{mm}^{2}$ $y$ debe encontrarse entre ciertos valores límite para evitar las fallas que puedan originarse por exceso o por defecto.

\section{Control del Proceso}

El objetivo de un control estadístico del proceso es hacer predicciones del mismo conforme al tiempo. Las gráficas de control son herramientas utilizadas para distinguir causas de variación en el proceso que son ajenas al natural desenvolvimiento del proceso.
Estas son utilizadas para detectar productos defectuosos o para indicar resultados de una modificación en los procesos, así como también informar a la gerencia repercusiones favorables en la producción.

Los productos o servicios que se producen en un proceso no serán iguales exactamente, debido a que siempre habrá muchas fuentes de variación, que nada puede hacerse para suprimirlas. Sin embargo, habrá para la gerencia opciones de investigación, para minimizar esas causas.

En la figura (Figura 4) se muestra como determinar la variación de un proceso. Se toman muestras y el valor se coloca en una escala proporcional en el eje $\mathrm{X}$, después en el eje vertical, se indica el número de veces que ocurrieron, es decir la frecuencia.

a) Las muestras varían de una a otra.

b) Forman un patrón estable.

c) La distribución difiere en la medida de la tendencia central.

d) Solo se presentan causas de variación naturales.

e) Se presentan causas de variación asignable $\mathrm{y}$ el proceso no es estable.

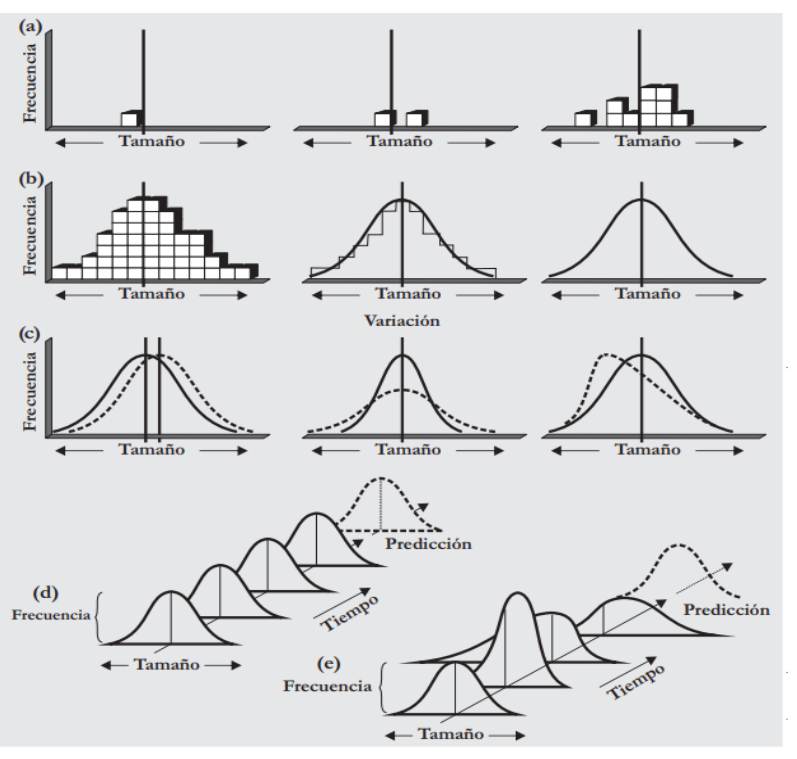

Figura 4 Diagramas para determinar la variabilidad de un proceso

Será trabajo del administrados de la producción mantener el proceso estable eliminando las causas asignables.

MEDINA-MENDOZA, José Antonio, MÉNDEZMACÍAS, Gerardo y HERRERA-REYES, Naieli. Análisis de la resistencia de piezas soldadas por el proceso de resistencia eléctrica. 2020 
La imagen siguiente (Figura 5) muestra los comportamientos diferentes de los procesos productivos en los que se muestran comportamientos anormales.

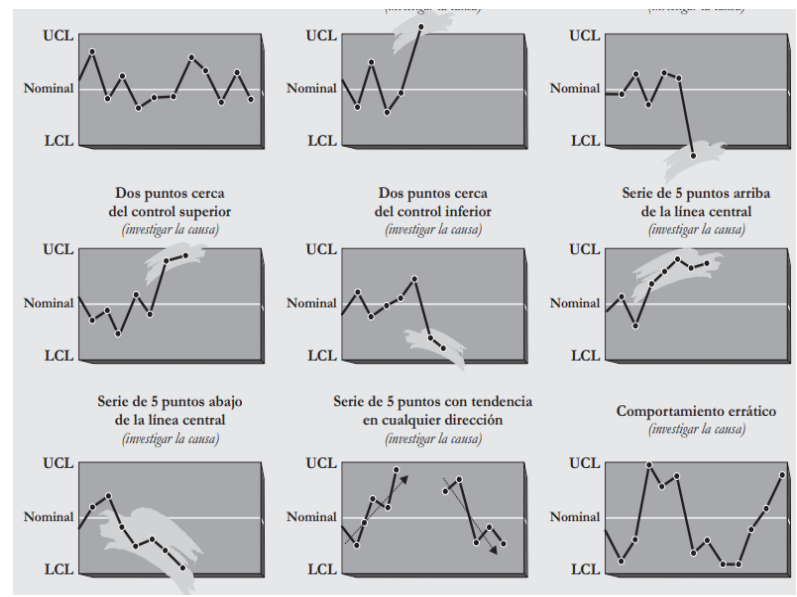

Figura 5 Comportamientos de los procesos productivos

\section{Metodología}

En el área denominada Automotriz de la empresa metalmecánica, trabajan cinco máquinas manuales de soldadura por resistencia eléctrica, las cuales producen partes con tuercas o tornillos soldados para formar soportes de uso automotriz.

Las máquinas son identificadas con numeración designada por el departamento de producción, para tener un control de estas tanto para su proceso como para el mantenimiento requerido.

La designación del número de máquinas que se han revisado para el presente estudio son las siguientes: máquina SW-6, máquina 205128, máquina 205-109, máquina 205-127 y máquina 205-110, las cuales pueden producir cualquiera de los productos que se manejan en la empresa, realizando los cambios requeridos para tal fin.

Hay dos variables que, debido a la normatividad aplicable, se cuidan dentro de los procesos de soldadura en esta empresa y son la resistencia a la tensión de la soldadura de tuercas y tornillos, así como, la resistencia a la torsión. Para lo cual se ha especificado que se deben de realizar pruebas de campo y obtener datos para revisar si se está cumpliendo con las normas establecidas.
Las pruebas de campo se realizan en una prensa marca POISE modelo DX-25. Las pruebas que se realizan son pruebas destructivas de tensión $\mathrm{y}$, por normatividad interna de la empresa, se deben de realizar cada dos horas (Figura 6).

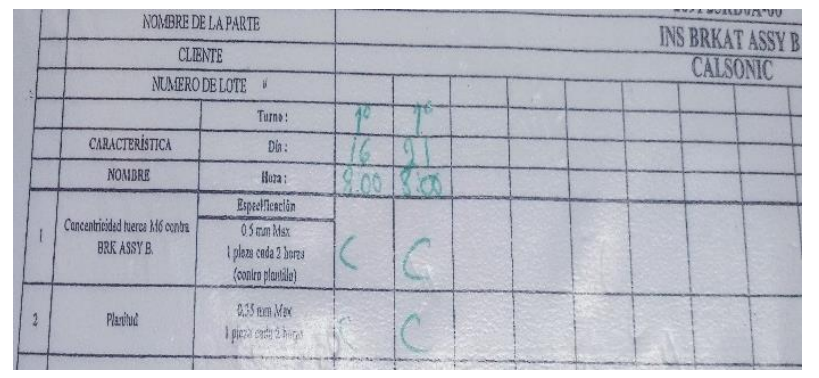

Figura 6 Norma establecida una pieza cada dos horas

La especificación establecida para los operarios está mostrada en una hoja de información anexa en la máquina de prueba y que informa la lectura mínima de resistencia que debe tener la pieza soldada (Figura 7).

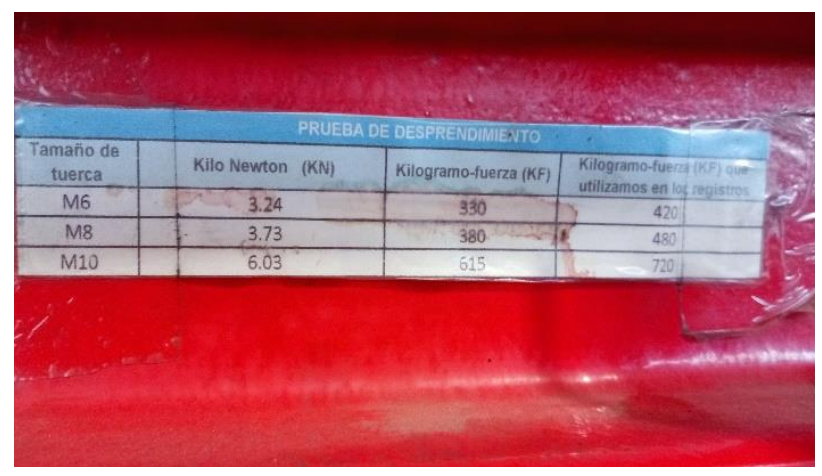

Figura 7 Norma mínima de resistencia por tamaño de tuerca

Los datos que se muestran en la figura 7 son obtenidos de la norma que rige este tipo de productos, que es la norma JIS B1186 2010 pagina 11 (Figura 8).

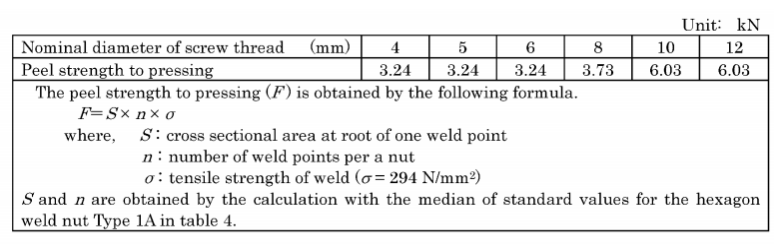

Figura 8 Norma JIS 11862010 (página 11)

Los datos obtenidos en la prueba de tensión son registrados en formatos específicos (Figura 9) para llevar el control de calidad correspondiente, siendo esta la fuente de donde se tomaron los datos para realizar el estudio de la variabilidad del proceso en cada una de las máquinas. 
Los datos se miden y registran en Kgf, debido a que es la escala de medición con la que cuenta este equipo.

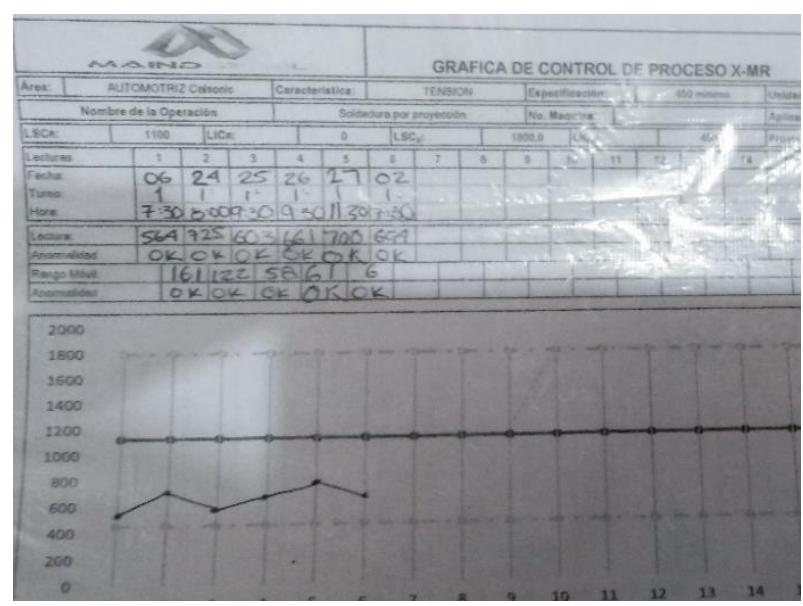

Figura 9 Registro de datos

La otra variable importante que se controla dentro del proceso es la resistencia a la torsión, para esta se cuenta con un dispositivo de diseño propio de la empresa (Figura 10 y Figura 11) y se le nombra solamente como poka yoke en donde se prueban $100 \%$ las piezas que tienen soldadura con tuercas M6.

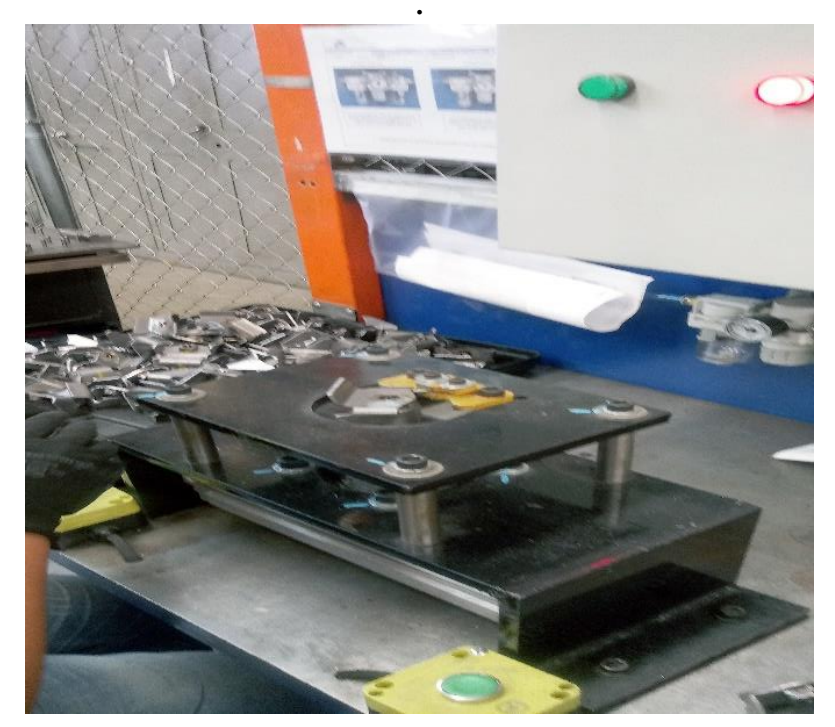

Figura 10 Poka yoke para prueba de torsión

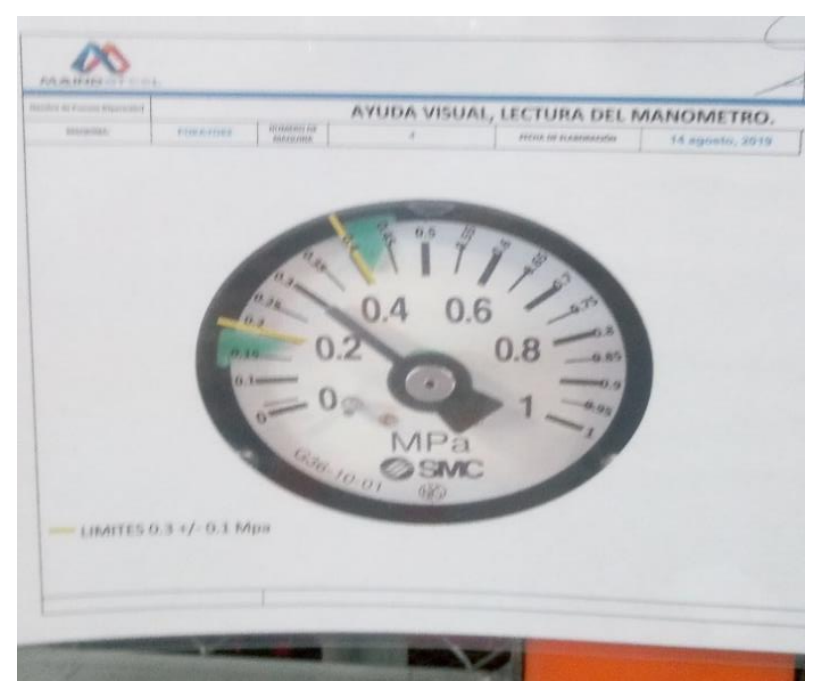

Figura 11 Ayuda visual para el ajuste de la fuerza de torsión en el poka yoke

$\mathrm{Al}$ realizarse la prueba se monta la pieza en el dispositivo y se le aplica una fuerza de giro por medio del uso de la neumática, equivalente a lo que marca la norma respectiva (Figura 12). Los valores para realizar la prueba se toman de la norma que rige este tipo de productos, que es la norma JIS B1186 2010 pagina 13.

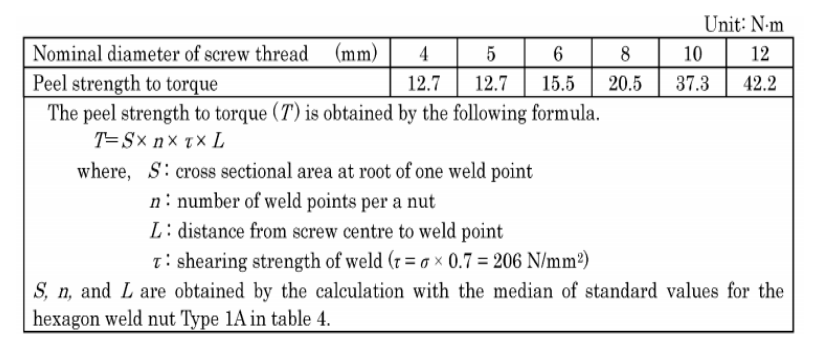

Figura 12 Norma JIS B1186 2010 (página 13), para pruebas de torsión en tuercas soldadas

En esta prueba no se registran los datos, solo se realiza para garantizar que las tuercas no se desprendan en el ensamble final en el automóvil.

\section{Resultados y conclusiones}

Los datos obtenidos en cada una de las máquinas fueron analizados mediante el programa $\mathrm{R}$ y nos muestran los siguientes resultados para cada una de ellas: 
Máquina SW-6:

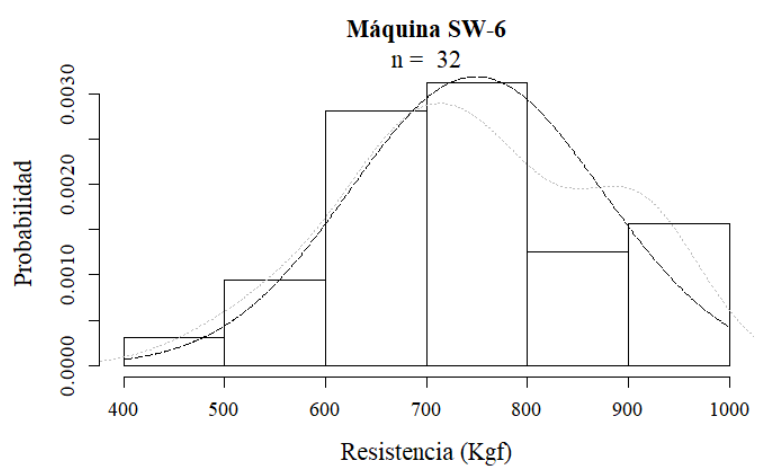

Gráfico 1 Histograma de frecuencia de máquina SW-6. La línea discontinua representa la distribución normal, la línea punteada representa la densidad empírica de los datos

Máquina 205-128:

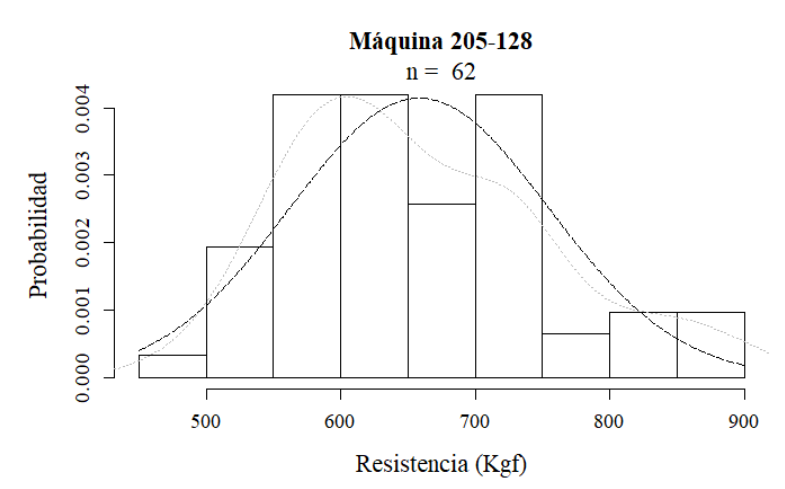

Gráfico 2 Histograma de frecuencia de máquina 205-128. La línea discontinua representa la distribución normal, la línea punteada representa la densidad empírica de los datos

Máquina 205-109:

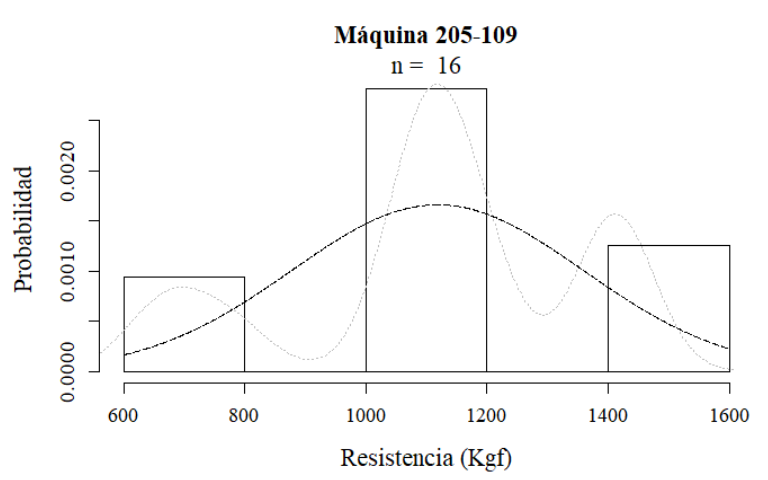

Gráfico 3 Histograma de frecuencia de máquina 205-109. La línea discontinua representa la distribución normal, la línea punteada representa la densidad empírica de los datos
Máquina 205-127:

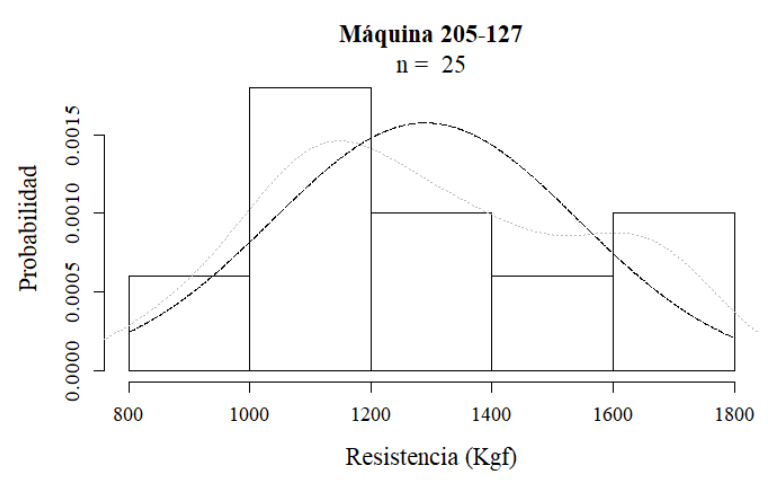

Gráfico 4 Histograma de frecuencia de máquina 205-127. La línea discontinua representa la distribución normal, la línea punteada representa la densidad empírica de los datos

Máquina 205-110:

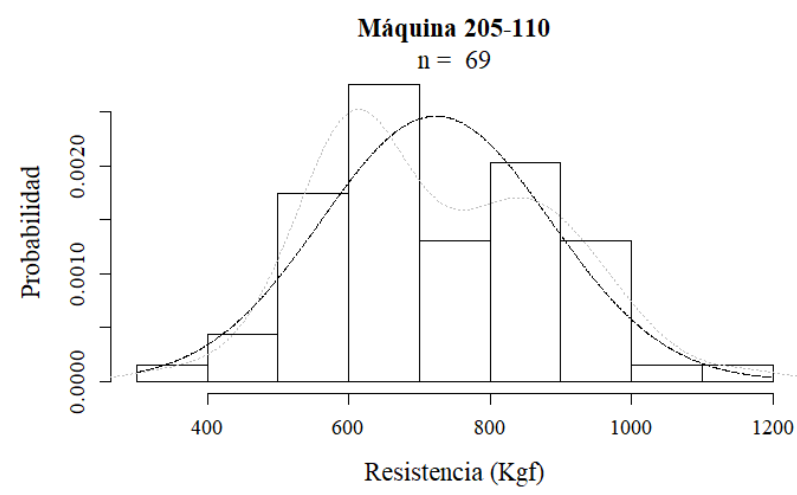

Gráfico 5 Histograma de frecuencia de máquina 205-110. La línea discontinua representa la distribución normal, la línea punteada representa la densidad empírica de los datos

\section{Datos estadísticos.}

En la Tabla 1 se muestran los datos estadísticos de cada una de las máquinas analizadas.

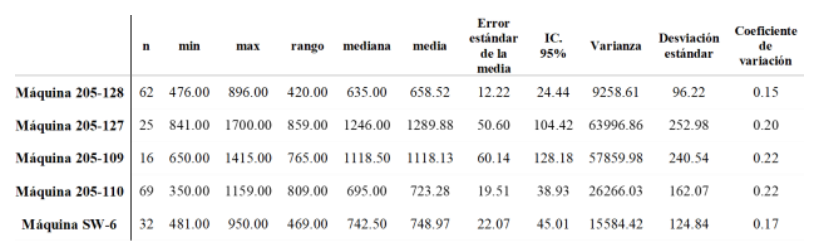

Tabla 1 Datos estadísticos

De acuerdo con los resultados, se concluye que la dispersión de los datos es muy amplia en todas las máquinas, por ejemplo, en la 205-127 el rango es de $859 \mathrm{Kgf}$, mientras que en la máquina SW-6 es de 420 Kgf. Aunque son diferentes tuercas las que se manejan, siendo la primera una M10 y la segunda una M6 en los dos casos es amplia de acuerdo con su rango. 
Se puede concluir también que no cumple con la realización de pruebas de torsión (Figura 13), de acuerdo a la norma JIS relacionada; no se cumple con la norma interna de realizar pruebas cada dos horas, solo se realizan dos, pero por turno.

\section{Recomendaciones.}

Con base en los resultados obtenidos, es recomendable que se ajusten los rangos para ser más cerrados y mejorar el control de la calidad de las piezas manufacturadas y, que, a la vez de cumplir su norma de resistencia mínima, no se pasen a una zona del límite superior en donde se vaya a fragilizar el ensamble por afectaciones de la soldadura.

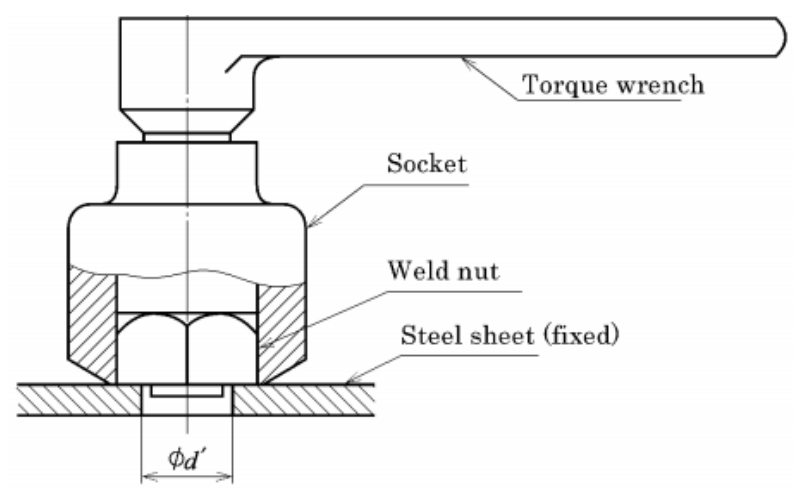

Figura 13 Diagrama de prueba de torsión

Rangos recomendados por máquina:

Ajustar las máquinas SW-6 y 205-110 a partir de la media encontrada en la muestra de más datos 658.52 con una tolerancia de $+/$ - de $100 \mathrm{Kgf}$.

Ajustar las máquinas 205-109, 205- 127 y 205-128 a partir de la media en la muestra de más datos 723.38 con una tolerancia de +/- 100 Kgf.

Realizar las pruebas de torsión según lo refiere la norma JIS relacionada para tener datos confiables de que se está cumpliendo, con base en JIS B1186 2010 (página 14).

Realizar las pruebas de campo según lo establecido para tener un mejor control de la calidad de las partes manufacturadas, ya que solo se toman dos datos, uno al iniciar el turno y otro a medio turno, debiendo de ser al menos 4 datos de prueba que deberían ser.
Se observó que los operarios que realizan las pruebas y toma de datos son los ayudantes de los operadores de las máquinas, que no dejan de trabajar mientras estas se desarrollan, en caso de que no se cumpla la resistencia mínima el operador para y cambia los parámetros de operación, generalmente modificando la corriente eléctrica de trabajo. Una vez que hace las modificaciones sigue trabajando, pero no da el tiempo para que el ayudante verifique una lectura de resistencia favorable, que en ocasiones no se cumple y hay que parar de nuevo para seguir modificando, pero en ese lapso ya pasaron piezas que están fuera de norma de resistencia y no se toma alguna medida al respecto. El operador deberá parar su máquina hasta estar seguro de que las piezas cumplen con la norma de resistencia.

\section{Referencias}

1.- F. Padilla 1, P. Gutiérrez C. 2, J. López G.3, (2015), Nueva industria regional en Aguascalientes. AMECIDER - CRIM, UNAM.

2.- Meseguer V J. L. 1; F. Chumillas P. 2; Serna S.J. 3; 18th International Congress on Project Management and Engineering Alcañiz, 16-18th July 2014.

3.- H. Zhang, J. Senkara. (2006). "Resistance Welding. Fundamentals and Applications". Taylor \& Francis Group. Florida, pp.1-17.

4.- American Welding Society, (2012). "AWS C1.1: Recommended practices for resistance welding" International Standard Book Number: 978-0-87171- 803-7.

5.- O. Martín**, M. López** y F. Martín** Redes neuronales artificiales para la predicción de la calidad en soldadura por resistencia por puntos. REVISTA DE METALURGIA, 42 (5) SEPTIEMBRE-OCTUBRE, 345-353, 2006 ISSN: 0034-8570

6.- J.Andradea*, Y. Ricob, Caracterización microestructural de la soldadura por electropunto en láminas de acero aisi 1015 galvanizado, Acta microscópica Vol. 27, No. 1,2018, pp 8-15 